\title{
EL TRABAJO DE LAS MUJERES EN EL SISTEMA ALIMENTARIO
}

\author{
Alicia Langreo Navarro ${ }^{\text {*, Tomás García Azcárate }}{ }^{\mathrm{b}}$ \\ a Directora de Sabora, S.L. E-mail: alangreonavarro@gmail.com ${ }^{\underline{b}}$ Vice-Director del Instituto de \\ Economía, Geografía y Demografía (IEGD-CSIC) e investigador asociado del CEIGRAM, Madrid, E- \\ mail: tomasgarciaazcarate@gmail.com.
}

Resumen: Nos referimos como sistema alimentario el conjunto de empresas cuyo concurso es imprescindible para la producción de alimentos. Abarca la producción primaria, la comercialización mayorista en origen y destino, la industria de primera y segunda transformación e incluso podría incluirse la distribución minorista; además del suministro (y en su caso fabricación) de insumos, los servicios logísticos, la asesoría en sus múltiples versiones o los servicios públicos vinculados. Anteriormente, hemos defendido que el sistema alimentario es el principal motor socioeconómico en la mayor parte de las áreas rurales. Aquí hacemos una aproximación de su aportación (en términos de PIB, ocupación, inversión y compras de materias primas) a los territorios españoles con problemas poblacionales (caída de la población, baja densidad, desequilibrios demográficos), para lo que utilizamos diversas fuentes estadísticas: Encuesta Industrial, Estadísticas agrarias, Padrón y Censo de la Población.

El ámbito geográfico y la cadencia condicionan el manejo de estas fuentes; se utiliza tanto la comunidad autónoma, como la provincia y, para la variable de ocupación, el rango del municipio según dimensión poblacional. Las limitaciones estadísticas no permiten contemplar los servicios al sistema alimentario ni los insumos, pero si la aportación de las fases primaria, la comercial mayorista y la industrial.

Palabras claves: Sistema alimentario, mujeres, mundo rural

\section{Introducción y metodología}

El número de mujeres ocupadas en el sector agrario que aparecen en la EPA o en el Censo de la Población es muy bajo y los datos no son mejores en los municipios más pequeños, donde el sector es más importante. Incluso las líneas de apoyo específicas para mejorar la posición de las mujeres presentan en general pobres resultados. Sin embargo, en nuestra opinión esa no es la realidad y la producción de alimentos cada vez cuenta con la participación de más mujeres, muchas de ellas cualificadas. En esta comunicación vamos a hacer una primera aproximación, entroncando con otros trabajos desarrollados por los autores sobre sistema alimentario, medio rural, la participación de las mujeres y las posibles actividades en la España vaciada. Este es un campo de trabajo abierto, en el que vamos a utilizar conceptos de los que no tenemos definiciones estadísticas ni, por lo tanto, información, por eso lo que presentamos aquí tiene como objetivo abrir un ámbito de trabajo y apuntar posibles vías de investigación.

\section{El punto de partida}

Planteamos el tratamiento conjunto del sistema alimentario lo que, en nuestra opinión, tiene un alto nivel de coherencia: las empresas de las fases integradas elaboran un único producto final, están altamente verticalizadas (mediante mecanismos de penetración de capital de una fase en otras, de absorción de unas fases por otras, de cesión de elementos de gestión, de acuerdos contractuales complejos y a medio plazo), la incorporación de innovaciones y la asunción de las señales del mercado se desplaza verticalmente entre las fases; esta cohesión interna se ha visto reforzada por la aplicación de la seguridad alimentaria (trazabilidad) y por los mecanismos de coordinación con la gran distribución minorista.

Además, el sistema alimentario es el más disperso geográficamente, debido a su estrecha vinculación con el sector primario, cuyo tratamiento industrial y adecuación comercial requiere proximidad. La única aproximación posible de evaluar lo que aporta la agricultura y lo que arrastra en términos de producción o de empleo en el territorio es la consideración conjunta de las fases del sistema alimentario e incluso de las restantes actividades que arrastra. Ahora bien, las fuentes estadísticas disponibles se organizan en base a los sectores económicos clásicos más o menos desagregados, lo que supone una dificultad enorme para desarrollar este enfoque.

Por otro lado, desde el último tercio del siglo XX ha cambiado profundamente el modo de producir, la producción de un bien se fragmenta entre empresas y áreas geográficas, se subcontratan tareas no esenciales en un principio y posteriormente se amplia este proceso y cobran más peso las asesorías externas de todo tipo, cada vez más necesarias según se sofistica y moderniza la estructura productiva. Este es un proceso general de la economía que en el caso del sector agrario está agravada por la pequeña dimensión de una buena parte de las empresas, que dificulta enormemente la modernización, la innovación y la gestión adecuada del trabajo y está generando que en muchas explotaciones al menos una parte considerable de las tareas fundamentales e incluso la gestión se realicen con contratos a terceros, fenómeno este muy mal recogido en las fuentes estadísticas. 
Para aproximarnos a la aportación de la agricultura y lo que arrastra en empleo, el primer paso es acotar el sistema alimentario. Hay un claro "núcleo duro" compuesto por la agricultura, ganadería y pesca en el sector primario, la industria alimentaria de primera y segunda transformación, el comercio mayorista en origen y destino de productos agrarios (materias primas) y alimentos y el comercio mayorista de animales vivos. Existe la discusión de si considerar o no en este núcleo a la distribución minorista de alimentos, en este trabajo no la vamos a considerar ya que en el análisis del medio rural encaja mejor como servicios. Ahora bien, las cosas se complican cuando realmente se amplia el objetivo a recoger el conjunto de actividad que arrastra el sistema alimentario; los ámbitos económicos que habría que incluir serían: el suministro de insumos a los sectores primarios, la industria o el comercio mayorista, las actividades de asesoría, gestoría, asesoría, certificación, servicios, logística, reparaciones y mantenimiento, etc.

Para medir la participación de las mujeres en el sistema alimentario se ha elegido es concepto "ocupación" del Censo de la Población y de la EPA, ambas del INE; en la primera la información se ofrece desagregada por sectores y subsectores, dimensión de los municipios y tipo de ocupación. Esta comunicación no permite una gran profundización en los distintos niveles, pero vamos a centrarnos en parte de las actividades del núcleo principal del sistema alimentario tanto en el conjunto de la economía como de los municipios de menos de 20.000 habitantes; esa estratificación hace imprescindible recurrir al Censo de la Población (lamentablemente el último de 2011). Asimismo, lanzaremos una discusión sobre los contrastes entre las cifras del Censo de la Población y el Censo Agrario \& Encuesta de Estructuras.

\section{La ocupación de las mujeres en la agricultura}

La información proporcionada por el Censo de la Población 2011 muestra un nivel de ocupación bajo de las mujeres en la agricultura tanto a escala nacional (4,46\% de los/las ocupados en España, el 5,75\% en hombres y el 2,9\% en mujeres) como en los municipios de menos de 20.000 habitantes $(9,21 \%$ de ocupación total, $11,5 \%$ en hombres y $6 \%$ en mujeres). Estas cifras son coherentes con la EPA (Encuesta Población Activa) 2019: 4\% del total de ocupados lo son en agricultura, el 5,7\% en hombres y el 2\% en mujeres. En términos absolutos, para 2011 había 230.365 mujeres ocupadas en agricultura en España y casi 136.000 en los municipios de menos de 20.000 habitantes. Hay que reseñar también que entre 2001 y 2011 cayó la ocupación de las mujeres en la agricultura, ganadería, silvicultura y pesca más del $28 \%$. Además la ocupación en agricultura es muy inferior en los tramos de menor edad: sólo el 3,7\% en las menores de 30 años, $10 \%$ entre 50 y 65 años y más del $16 \%$ por encima, lo que augura un mal futuro.

Las estadísticas agrarias (Censo Agrario y Encuesta de Estructuras Agrarias) recogen conceptos distintos. En 2009 (fecha del último Censo Agrario publicado) 1.089.902 personas vinculadas a la explotación familiar $^{24}$, entre ellas 474.724 mujeres (44\%), ahora bien, la inmensa mayoría tanto de hombres como de mujeres tenían una dedicación mínima ( $83 \%$ de media dedican menos del $25 \%$ del tiempo de trabajo a la explotación). Únicamente 82.213 personas dedican más del $50 \%$ del tiempo de trabajo anual a la explotación, entre ellas 33.237 mujeres.

Por otro lado, entre los trabajadores fijos en el año del Censo agrario se contaban más de 120.000 hombres con más del 50\% del tiempo de trabajo frente a unas 23.000 mujeres. Es decir, en 2009, entre las mujeres vinculadas a la explotación familiar y las asalariadas fijas sumaban menos de 60.000 personas con una dedicación superior al 50\%. Con respecto a los asalariados eventuales, el INE sólo ofrece datos del número de horas trabajadas anualmente en total, ni el número de personas que las realizan ni su sexo, sin embargo cabe mencionar que la presencia de mujeres entre los asalariados eventuales es pequeña y disminuye rápidamente, manteniéndose de forma significativa en tareas muy específicas.

Estas cifras muestran una escasa vinculación de las mujeres con el trabajo en la agricultura, ¿qué hay detrás? Para aproximarnos a una respuesta es necesario bucear en las tarea desarrolladas en la agricultura y la ganadería, en como se hacen, quien las hace, y como han evolucionado ${ }^{25}$. La información más completa, aunque anticuada se encuentra en el informe realizado por Saborá y subvencionado por la FORCEM en

\footnotetext{
${ }^{24}$ Estas cifras sólo se refieren a las explotaciones cuyo titular es una persona física, quedan fuera las Comunidades de Bienes, Cooperativas, sociedades mercantiles, etc.

${ }^{25}$ No hemos encontrado análisis socioeconómicos académicos al respecto, pero si referencias en los convenios colectivos del campo e interesantes análisis desde los ámbitos de investigación en maquinaria y modernización de las tareas agrarias, aunque sin apenas referencias de género.
} 
1997-9826; en él se hizo una recopilación bastante exhaustiva de las tareas realizadas por mujeres en agricultura y ganadería siendo las principales conclusiones: los grandes cultivos herbáceos estaban totalmente mecanizados y en ellos no trabajaban mujeres ${ }^{27}$, en los frutales la participación de las mujeres caía y se centraba en la recogida (tarea de corta duración), pero ya entonces estaban siendo sustituidas por inmigrantes, pasando ellas a trabajar prioritariamente en las centrales hortofrutícolas (fuera del sector agrario), en la viña y el olivar quedaba trabajo de las mujeres en las recolecciones manuales y en la viña en la poda en verde, la caída de la participación femenina está vinculada a la mecanización y la participación de inmigrantes; en las hortalizas para industria y algunas de invierno, que ya tienen recolección mecanizada, quedan también pocas mujeres participando en las tareas, algo mayor es la participación en la huerta para fresco, pero también aquí la participación femenina se está desplazando a las centrales hortofrutícolas.

Queda una participación importante en las tareas agrícolas en subsectores donde se considera que las mujeres lo hacen mejor (flores, semilleros, fresas y otros frutos rojos y demás productos delicados), así como en determinadas ganaderías, de leche, cría y algunos animales pequeños. También destaca la participación de mujeres en agricultura ecológica, esta vez vinculada a la propiedad de la explotación. La mayor parte de las mujeres que realizaban tareas agrarias y/o ganaderas estaban vinculadas a la explotación familiar, generalmente como ayuda familiar y colaboraban en tareas de corta duración.

Todo esto explica que las cifras de ocupación de las mujeres en el sector agrario sean bajas. Por otro lado en las estadísticas estructurales del sector (Censo Agrario y Encuesta de Estructuras de las Explotaciones Agrícolas, ambas del INE) aparece una cifra de mujeres titulares de explotación que se sitúa ligeramente por encima del 30\%, con una tendencia al alza; este hecho, junto a la pequeña dimensión de la mayor parte de estas explotaciones, se remarca en múltiples informes y trabajos académicos, pero hay que tener en cuenta que en las explotaciones muy pequeñas la titularidad apenas implica dedicación, hay que recordar que la mayor parte de ellas tienen menos de una UTA (unidad de trabajo anual). ${ }^{28}$ En el Censo Agrario de 1989, únicamente el $23 \%$ de los titulares de explotación eran mujeres, de estas el $76 \%$ en explotaciones con menos de 5 hectáreas. Las cosas han cambiado, aunque se esté lejos de una participación equilibrada, en la última Encuesta de Estructuras (2016), las mujeres suponían el 32,4\% de los titulares de explotación, el $57 \%$ en explotaciones menores de 5 hectáreas.

La escasa presencia de mujeres en la dirección de explotaciones de una cierta dimensión es la causa directa de que apenas se encuentren en las asambleas y consejos rectores de las cooperativas y en las organizaciones agrarias. En el estudio mencionado se encontró una participación de menos del 5\% en las Asambleas de las cooperativas y realmente insignificante en los consejos rectores. En las OPA la participación de mujeres se centraba en las secciones o asociaciones específicas de mujeres. Las cosas han mejorado desde entonces debido de una parte a la mejor posición en las explotaciones y de otra a la tarea desarrollada tanto por las Cooperativas, las Organizaciones Agrarias y las Administraciones públicas para avanzar en la igualdad. No tenemos datos actualizados de las OPA, pero según la Confederación de Cooperativas Agroalimentarias, en 2018 la participación de mujeres como socias había subido al 26\%, en los Consejos rectores había un $7,4 \%$ de mujeres y las mujeres presidentas suponían un 3,6\%, es una mejora notable.

\section{La ocupación de las mujeres en el resto del sistema alimentario}

En la industria alimentaria y de bebidas reúnen el 3,57\% de las mujeres ocupadas y en el comercio mayorista el 3,2\% (estimación), lo que sumado al 6\% de la agricultura (incluida ganadería, pesca y silvicultura) alcanza el 12,81\% de la ocupación de las mujeres en los municipios de menos de 20.000 habitantes (2011). Hay que resaltar que la ocupación en toda la industria está cayendo. Para el conjunto de España las mujeres ocupadas en agricultura están por debajo del $3 \%$ y en la industria alimentaria apenas superan el $2 \%$.

\footnotetext{
${ }^{26}$ Saborá SL (1997-98) La formación para la mejora de la posición de las mujeres en el sector agrario. 1997-98. Estudio financiado por la FORCEM y realizado con la colaboración de ASAJA, COAG, UPA, CCAE, FECAMPO-CCOO y FTT-UGT. Incluye un trabajo de campo en 40 comarcas y en todas las CCAA que tenía entre sus fines identificar las tareas realizadas por las mujeres.

${ }^{27}$ En todos los sectores se observa que no hay mujeres manejando la gran maquinaria (camiones, maquinaria de obras públicas, maquinaria agrícola, puertos, etc.).

${ }^{28}$ A pesar de que la mayor parte de titulares de explotación lo sean de explotaciones pequeñas, ya en el Libro Blanco de la Agricultura y el Desarrollo Rural (MAPA 2003) se detectó que a finales del siglo XX había aumentado significativamente el número de mujeres titulares de explotaciones situadas en los estratos de mayor dimensión económica. Esa tendencia ha continuado.
} 
Hasta aquí es lo que permiten las estadísticas disponibles. Pero hay muchas más mujeres aportando su trabajo en ámbitos imprescindibles para el funcionamiento del sistema alimentario:

- Según la Confederación de Cooperativas Agroalimentarias es muy significativo el número de mujeres en puestos técnicos, de control de calidad y de gestión en las cooperativas y está aumentando.

- Hay un número muy significativo y creciente de mujeres como técnicos en asociaciones de defensa sanitaria, en grupos de servicios veterinarios, en laboratorios de análisis vinculados al sector y en empresas de certificación.

- La presencia de mujeres en la industria alimentaria es dispar; por subsectores son numerosas por ejemplo en conservas de pescados, frutas y hortalizas, pero apenas se encuentran en mataderos, bodegas o almazaras. En general en todos los subsectores hay mujeres en puestos comerciales, en laboratorios, por supuesto en administración o contabilidad e innovación o control de calidad. Su posición ha mejorado notablemente en gestión; en este caso es mayor en las empresas pequeñas y medianas familiares.

- En las empresas comerciales de suministros a la agricultura o industria alimentaria, cuentan con mujeres en todo tipo de puestos de trabajo, excepto trabajo directo en almacenes y logística.

- Hay pocas mujeres en las empresas comerciales de animales vivos

- En las firmas mayoristas de productos agrarios la participación de mujeres depende mucho de los subsectores: abundan en las centrales hortofrutícolas pero apenas se encuentran entre los almacenistas de cereales o leguminosas.

- Apenas hay mujeres en las sociedades de servicios que desarrollan tareas en las explotaciones con contratos a terceros, ni en las de labores con maquinaria ni en las tareas manuales. Se encuentran mujeres en la externalización de la gestión.

\section{Conclusiones}

La ocupación de las mujeres directamente en la agricultura o en la industria alimentaria no es alta, ni en España ni en el medio rural. Sin embargo su aportación al sistema alimentario es muy importante y está creciendo en puestos de trabajo vinculados a la modernización y mejora del conjunto del sistema. Los escasos niveles de ocupación en agricultura contrastan con el mayor número de titulares de explotación, y se deben a su escasa participación en las tareas con maquinaria y su sustitución en la mayor parte de las tareas manuales. Las fuentes estadísticas no permiten medir la participación de las mujeres en el conjunto del sistema alimentario, pero es posible buscar datos parciales que permitan avanzar en el conocimiento de la situación, para eso habría que implicar a las asociaciones empresariales vinculadas al sector.

\section{Bibliografía}

Allen, P.; Sachs, C. (2007): Women and Food Chains: The gendered Politics of Food International Journal of Sociology of Food and Agriculture 15 (1): 1-23

Benito, I.; Langreo Navarro, A. (2009): Estrategias para la igualdad entre hombres y mujeres en la agricultura, el medio rural y el sistema alimentario Anuario de la Agricultura Familiar (122-132) Vicente-Mazariegos, J. ' 1989): Las mujeres en la agricultura europea: elementos para su estudio sociológico. Agricultura y Sociedad 51: 205-228 